\title{
Baha Attract Implantation Using a Small Incision: Initial Report of Surgical Technique and Surveillance
}

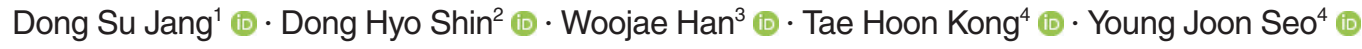 \\ ${ }^{l}$ Department of Sculpture, Hongik University, Seoul; ${ }^{2}$ Department of Fine Arts Education, Kyungnam University, Changwon; \\ ${ }^{3}$ Department of Speech Pathology and Audiology, Research Institute of Audiology and Speech Pathology, College of Natural Sciences, \\ Hallym University, Chuncheon; ${ }^{4}$ Department of Otorhinolaryngology, Yonsei University Wonju College of Medicine, Wonju, Korea
}

Objectives. To determine the appropriate anatomical borders of implantation on the temporal bone in a cadaver study, and to develop a simplified surgical technique for Baha Attract implantation through a small incision along the hairline using anatomical evidence and a navigation system.

Methods. In a cadaver study, 20 human adult dry skulls were used to find flat areas of the temporal bone for Baha Attract magnet implantation. Four borders of the "optimal surgical site" were defined: Asterion line, occipitomastoid suture line, sigmoid sinus line, and digastric groove line. In three patients, we implanted the Baha Attract according to the newly developed surgical procedure and validated the feasibility of this technique with a navigation system.

Results. We identified the appropriate position of the implant on the temporal bone, suggesting a simplified surgical technique for Baha Attract with a small incision. We determined the spot of implantation, and the implants were inserted through a small surgical incision $(<2.5 \mathrm{~cm})$ under local anesthesia; the procedure lasted approximately 30 minutes.

Conclusion. The optimal surgical site of the temporal bone is a safe and easily accessible location for implantation of the Baha Attract.

Keywords. Baha Attract; Surgical Procedures; Cadaver; Navigation

\section{INTRODUCTION}

Bone conduction implants are a very useful alternative for rehabilitating patients in cases where conventional hearing aids are not adapted or are unacceptable, such as in cases of single-sided deafness or conductive/mixed hearing loss [1]. The principle is that sound can be transferred to the inner ear by skull vibrations, bypassing the external and middle ear. Since the first implant was reported in 1977, more than 10,000 patients worldwide have received the bone conduction implants [2]. Despite this success, several shortcomings are known. Percutaneous

- Received March 13, 2019

Revised May 22, 2019

Accepted May 30, 2019

- Corresponding author: Young Joon Seo

Department of Otorhinolaryngology, Yonsei University Wonju College of

Medicine, 20 Ilsan-ro, Wonju 26426, Korea

Tel: +82-33-733-0640, Fax: +82-33-741-0644

E-mail: okas2000@yonsei.ac.kr abutment requires lifelong daily hygiene control. The possible complications include infection, skin overgrowth and, in some cases, loss of implants (range, $8 \%$ to $59 \%$ ), which can occasionally lead to revision surgery (range, $5 \%$ to $42 \%$ ) $[3,4]$. The aesthetic appearance is also a problem due to low-grade infections around the abutment, personal preference, and skin-penetrating implants behind the ear [5]. Several solutions have been proposed in which the skin remains intact [6-8].

A new system, the Baha Attract, was developed in 2013 [9]. A sound processor with a bone conduction transducer (vibrator) is attached to the outside of the intact skin. The sound processor is attached to a corresponding external magnet. Two magnetic discs are used: one under the skin connected to the implant, and a second external disc with a sound processor. A pad of soft material covers the surface of the magnet and distributes the pressure to the skin and soft tissue between the magnets. Surgery can be performed under local or general anesthesia and lasts from 40 to 80 minutes [10-12]. For satisfactory positioning, a

Copyright $\odot 2020$ by Korean Society of Otorhinolaryngology-Head and Neck Surgery

This is an open-access article distributed under the terms of the Creative Commons Attribution Non-Commercial License (https://creativecommons.org/licenses/by-nc/4.0)

which permits unrestricted non-commercial use, distribution, and reproduction in any medium, provided the original work is properly cited. 
large incision is used to insert the Baha Attract.

Here, we identified the appropriate anatomical position of the implant on the temporal bone in a cadaver study and developed a simplified surgical technique to implant the Baha Attract through a small incision $(2.5 \mathrm{~cm})$; we omitted the bone polishing that was used in three patients. We successfully implanted the Baha Attract according to the newly developed surgical procedure and validated the feasibility of this technique. The method developed has three advantages over the conventional technique: (1) improved cosmetic effect, as abnormal hair growth that occurs along a semicircular incision line does not occur when using this technique; (2) omitted polishing of the temporal bone; and (3) a short operating time ( $<30$ minutes), thus enabling the use of local anesthesia.

\section{MATERIALS AND METHODS}

\section{Cadaver study}

The study included 20 human adult dry skulls of an Asian population. The specimens were obtained from the Department of Anatomy at Yonsei University Wonju Severance Christian Hospital. Included skulls were injury-free. The skulls were excluded if a temporal bone was not intact or a history of head trauma was reported. The exact ages and sexes of the skulls were known. The average age was 64.5 years (range, 32 to 83 years) and the male to female ratio was 12:8. The skulls were studied to determine flat areas of the temporal bone for placement of the Baha Attract implant magnets. The conventional surgical site is anterior to the sigmoid sinus and requires bone polishing due to the temporal line (Fig. 1A). We determined that the optimal surgical site is appropriate for implant placement; it is located in the retrosigmoid position. The borders of the optimal surgical site are as follows: (1) the anterior border is a line of the sigmoid sinus (sigmoid line); (2) the posterior border is a line of the occipitomastoid suture (occipitomastoid suture line); (3) the superior border is a line from the Asterion to the temporal line (Asterion line); and (4) the inferior border is a line from the end of the digastric groove in parallel (digastric line) (Fig. 1B).

We calculated three parameters on each side of each skull: the distance from the spine of Henle to the Asterion, the distance from the spine of Henle to the sigmoid sinus in parallel, and the area of the optimal surgical site. Using transparent pa-

\section{H I G H L I G H T S}

- We identified the appropriate anatomical position of the Baha Attract on the temporal bone in a cadaver study.

- We also developed a simplified surgical technique through a small incision through a small incision $(2.5 \mathrm{~cm})$ under local anesthesia.
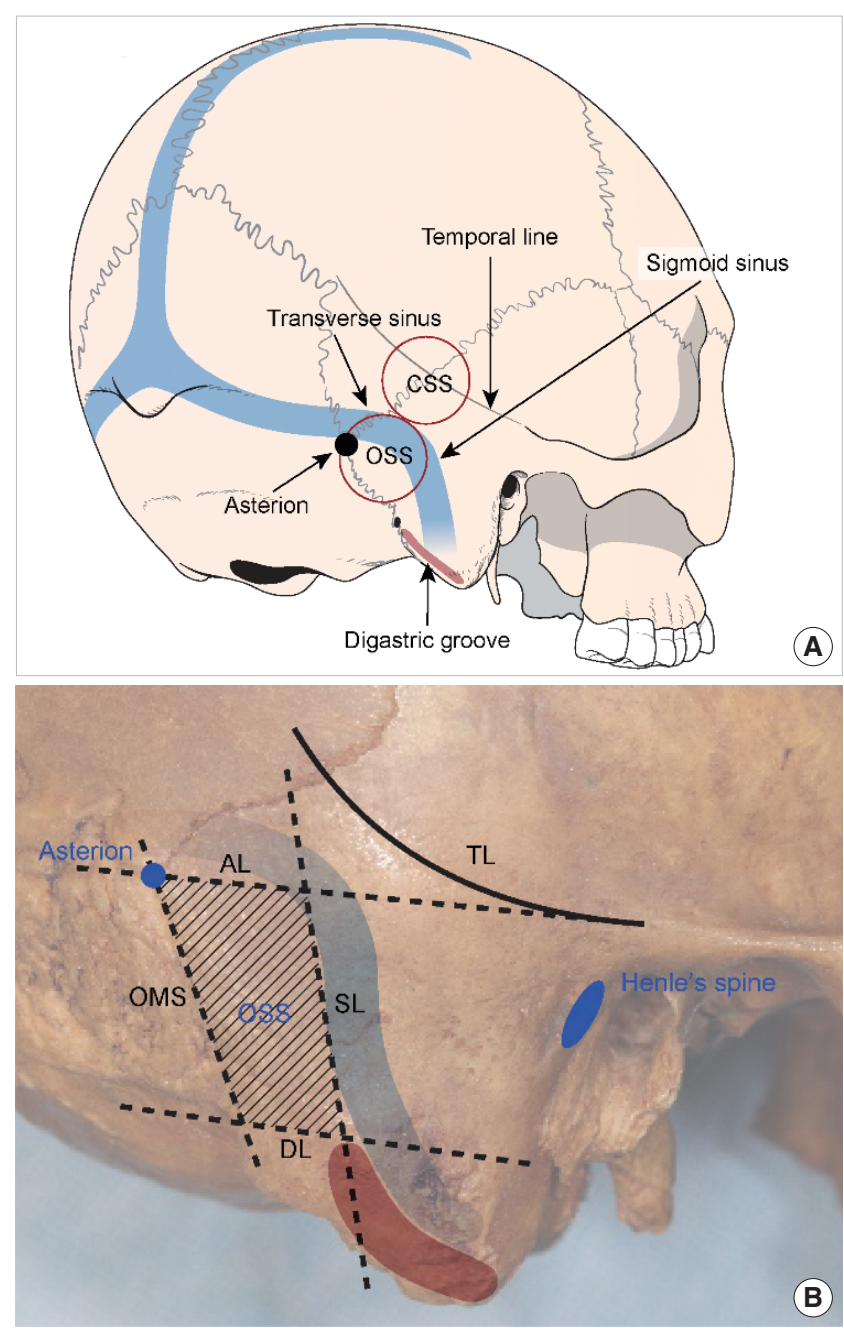

Fig. 1. (A) The anatomical distribution of the temporal bone shows that the optimal surgical site (OSS), with a relatively flat surface, is a better position for the Baha Attract implant. (B) The four borders of the OSS are the Asterion line $(A L)$, sigmoid sinus line (SL), occipitomastoid suture line (OMS), and digastric groove line (DL). The average distances from the spine of Henle horizontally to the Asterion and to the Sigmoid sinus are necessary to determine the location of the implant during surgery. As the size of the implanted magnet is $27 \mathrm{~mm}$, the area of the OSS should be sufficient. CSS, conventional surgical site; TL, temporal line.

pers positioned tightly on the temporal bone, we drew the optimal surgical site on the papers. We then analyzed the differences between the two groups.

\section{Preoperative surgical planning of navigation-assisted demarcations on three-dimensional computed tomography} Computed tomography (CT) datasets of the temporal bones ( $768 \times 768$ pixels; resolution, $0.129 \mathrm{~mm} /$ pixel; slice thickness, $0.67 \mathrm{~mm}$ ) were imported into the three-dimensional simulation software program in the Digital Imaging and Communications in Medicine (DICOM) format. The location of the optimal surgical site was determined in the cadaver study described in the 


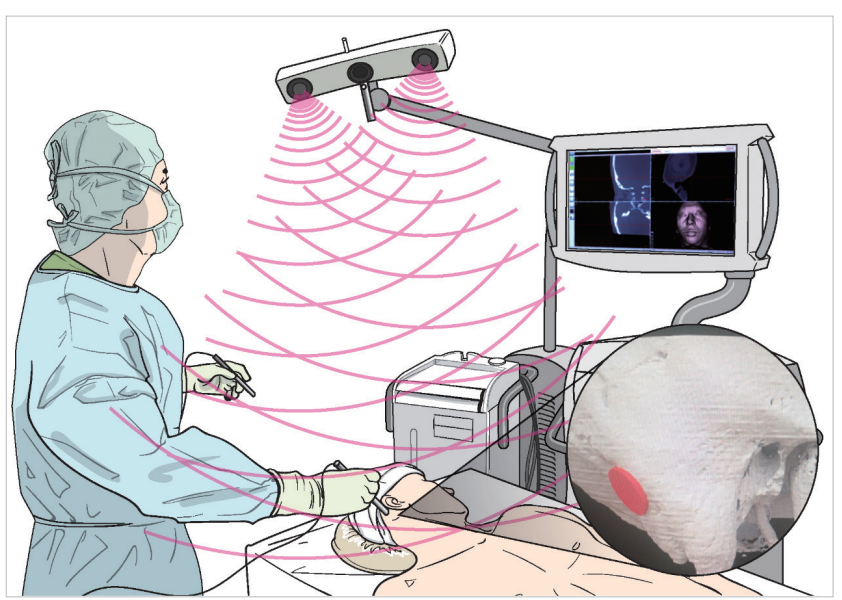

Fig. 2. The navigation system view during the implantation of Baha Attract. Just before the surgery, we marked the spot of the implant in the patient with the appropriate position measured in previous temporal bone computed tomography.

previous section. The optimal implant site was determined on CT images before surgery (Fig. 2). Before the patient entered the room, the patient's radiological data were transferred to the surgical navigation system workstation. We followed the protocols of the previous navigation study when using the navigation system, which was used in Bonebridge surgery [13]. The Scopis Hybrid Navigation System (Megamedical, Seoul, Korea) was developed for use in ENT surgeries and helps doctors recognize the direction and structure of the operating area and the exact location of surgical equipment. We marked the location of the optimal surgical site on the skin covering the temporal bone of each patient before the aseptic drape.

\section{Operative technique with a minimal skin incision}

Three patients received the implant under local anesthesia. Most steps followed the recommended procedures described in the company's surgery guide, with the exception of the skin incision line and polishing of the bumps of the temporal bones (Fig. 3). First, the lines of the imaginary temporal line, hairline, and the contours of the magnetic dummy were drawn. Then, the site of the linear incision, $20-30 \mathrm{~mm}$ in length and following the hairline, was determined. After measuring the skin thickness with a needle, a linear incision after a local anesthesia is made down to the periosteum. Next, dissection of the periosteum over the temporal bone was needed to ensure adequate space for the magnet. The template of the implant magnet was placed on the periosteum to ensure good positioning of the implant magnet. A cruciate incision $\left(6 \mathrm{~mm}^{2}\right)$ was made in the periosteum to expose enough bone for the implant flange. After drilling with the Guide drill and the widening drill on the planned spot, the implant was placed. We checked the thickness of the bone before surgery to ensure it would be adequate. No bumps were permitted around the implant in the flat optimal surgical site. The bone
Table 1. Patients' characteristics with Baha implantations

\begin{tabular}{lccc}
\hline Variable & Patient 1 & Patient 2 & Patient 3 \\
\hline Sex/age (yr) & Male/75 & Male/55 & Male/59 \\
Type of hearing loss & Mixed & Sensorineural & Mixed \\
Pre-PTA (air/bone conduction, dB) & $76 / 45$ & Deaf & $92 / 52$ \\
Site of surgery & Right & Left & Right \\
Anesthesia & Local & Local & Local \\
Surgery time (min) & 22 & 20 & 25 \\
Size of incision (mm) & 25 & 28 & 25 \\
\hline
\end{tabular}

PTA, pure tone audiometry.

bed indicator was checked and rotated to ensure it did not contact the bone. Before attaching the implant magnet, we confirmed with a soft tissue gauge that the flap was $6 \mathrm{~mm}$ thick. The flap was placed over the implant magnet and sutured through the periosteum. The incision line $1-2 \mathrm{~mm}$ from the magnet was checked.

Audiologic evaluation and surgery outcomes in three patients Three patients received Baha Attract implants, and they were followed up at our hospital for 6 months (Table 1). One month after the surgery, an external Baha 5 device was attached to the magnet. We evaluated audiologic function as well as the surgery site. The sound field set-up was calibrated according to Morgan et al. [12] Functional gains were calculated at the implant ear; with the contralateral ear with making. We primarily used the Hearing in Noise Test (HINT) to investigate speech discrimination in noise. We set speakers $1 \mathrm{~m}$ in front, to the right, and to the left of the patient. The signal-to-noise ratio or $\mathrm{dB}$ at which $50 \%$ speech discrimination or word recognition occurred was evaluated when the patient was presented with (1) a quiet situation, (2) sound from the front with noise from the right or the left side, (3) and noise from the front with sound from the right or the left side at speech levels similar to those in everyday life (65-75 dB) [14]. We also evaluated if the site of the implant was appropriate by examining the wound dehiscence, degree of scarring, disturbance of hair growth, and location of the implant.

\section{Ethical considerations}

This study was approved by the Institutional Review Board of Yonsei University Wonju College of Medicine (IRB No. 2016-110006). Written informed consent was obtained from all participants. All methods were performed in accordance with the relevant guidelines and regulations of our IRB.

\section{RESULTS}

\section{Identification of the appropriate position of the implant on the temporal bone}

Samples from 20 cadavers were divided into 20 right and 20 left sides, and the topographical distributions of the temporal bone 


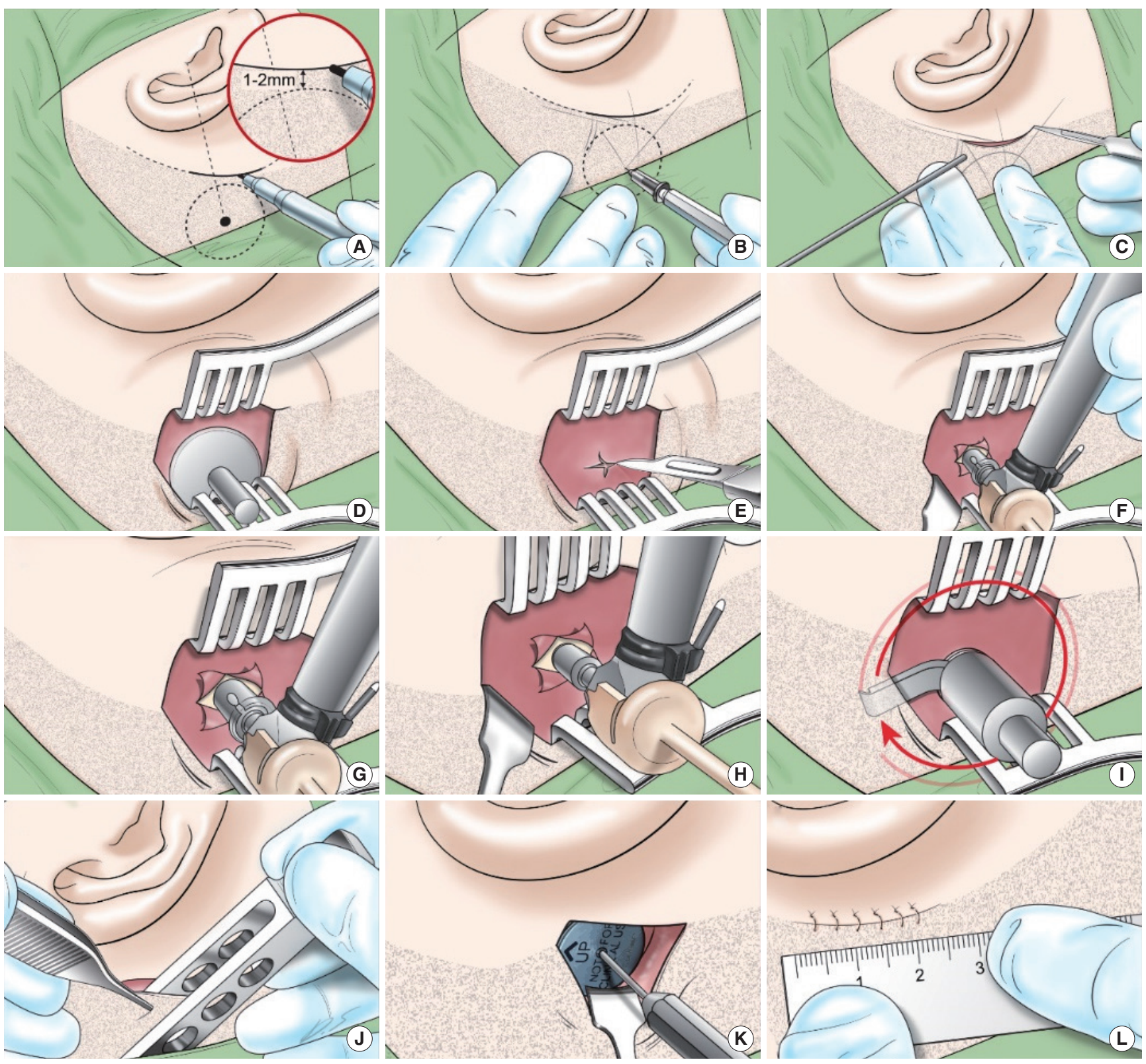

Fig. 3. The steps of the newly developed surgical procedure for implantation of the Baha Attract. (A) The small incision line along the patient's hairline was marked. (B) The soft tissue thickness was measured with a thin needle. (C) An incision was made down to the periosteum. (D) The implant magnet template was placed on the periosteum. (E) A cruciate incision was made on the periosteum, and the periosteum was lifted. (F) The bone was drilled with a guide drill to a depth of $4 \mathrm{~mm}$. (G) The hole was widened with the widening drill. $(\mathrm{H})$ The implant was placed with a torque of 40-50 N-cm. (I) The bone bed indicator was used to confirm that the implant did not touch the bone or periosteum around the implant. (J) The thickness of the flap was evaluated with the soft tissue gauge, and should be 3-6 mm. If the skin flap was thicker than the reference point, the flap was thinned to $6 \mathrm{~mm}$. (K) The implanted magnet was attached and screwed clockwise. (L) The skin was sutured to the periosteum over the implanted magnet. In the previous method provided by Cochlear Americas Corporation, a large C-shaped incision with a $150^{\circ}$ angle across the hairline remained. Due to the large flap, additional steps were necessary to find a flat area of bone and to avoid the suture lines in the bone.

were determined (Fig. 4). The optimal surgical site was marked according to the proposed boundaries. We calculated the distance from the spine of Henle to the sigmoid sinus horizontally, and the distance to the Asterion horizontally from the spine of Henle to determine the approximate position of the screw from the spine of Henle. The area of the optimal surgical site was measured to see if there was enough space for the implanted magnet.

There was no significant difference in any of the three indices between the values of the temporal bone measured on the right 

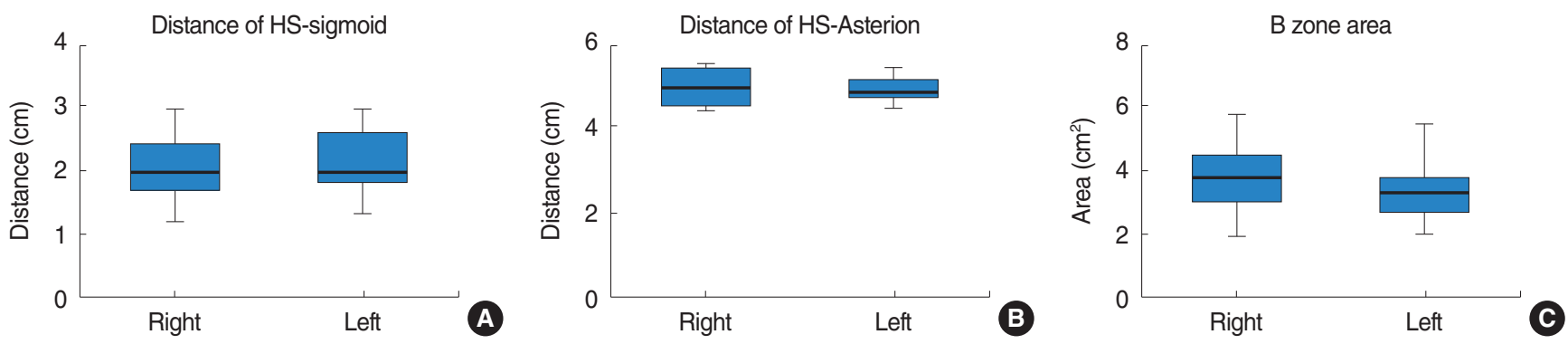

Fig. 4. Topographical distributions of the temporal bone. There was no difference between the right and left sides. (A) The distance from the spine of Henle (HS) to the sigmoid sinus (sigmoid) was $2.04 \pm 0.01 \mathrm{~cm}$ on the right side and $2.14 \pm 0.10 \mathrm{~cm}$ on the left side (P=0.499). (B) The distance from the HS to the Asterion was $5.05 \pm 0.09 \mathrm{~cm}$ on the right and $4.97 \pm 0.07 \mathrm{~cm}$ on the left $(P=0.479)$. (C) The area of the optimal surgical site was $3.74 \pm 0.24 \mathrm{~cm}^{2}$ on the right and $3.37 \pm 0.18 \mathrm{~cm}^{2}$ on the left $(P=0.225)$.
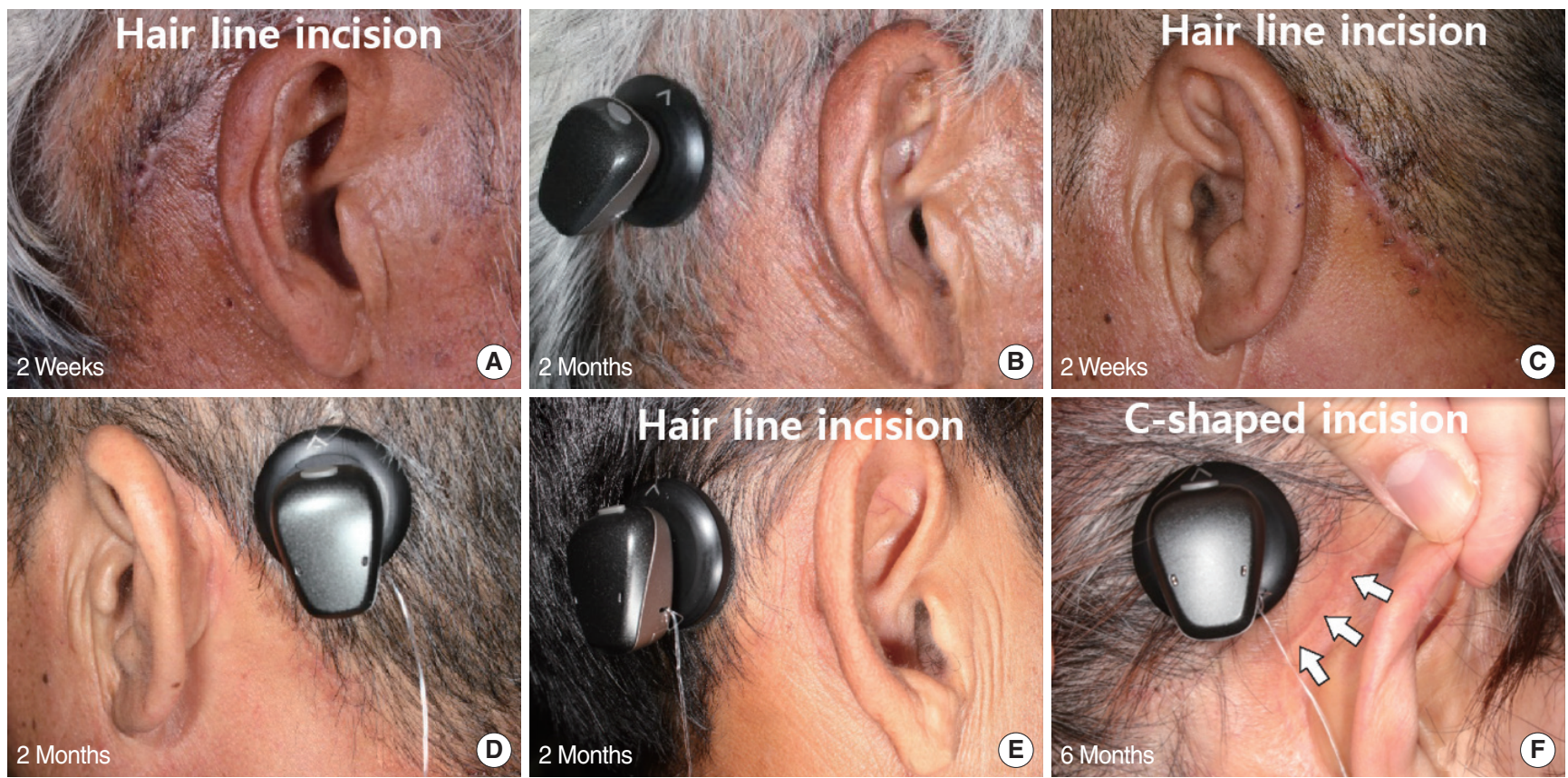

Fig. 5. The postoperative figures of the incision site. $(A)$ and $(C)$ show small incisions according to the hairlines of two patients 2 weeks after implantation. Patients in (B), (D), and (E) showed no scars in the postauricular area after 2 months. (F) The scar of a 63-year-old woman who received the implant through a large C-shaped incision, 6 months after implantation (white arrows).

and left sides. The distance from the spine of Henle to the sigmoid sinus was $2.04 \pm 0.01 \mathrm{~cm}$ on the right and $2.14 \pm 0.10 \mathrm{~cm}$ on the left $(P=0.499)$. The distance from the spine of Henle to the Asterion was $5.05 \pm 0.09 \mathrm{~cm}$ on the right and $4.97 \pm 0.07 \mathrm{~cm}$ on the left $(P=0.479)$. The area of the Optimal surgical site was $3.74 \pm 0.24 \mathrm{~cm}^{2}$ on the right and $3.37 \pm 0.18 \mathrm{~cm}^{2}$ on the left $(P=$ $0.225)$. A required diameter of the magnet is about $27 \mathrm{~mm}$.

\section{Development of a simplified surgical technique for Baha Attract with a small incision}

The results obtained from the cadaver study enabled simplified surgery using a small incision. The precise location of the optimal surgical site allowed us to select a safe, flat position for the magnet on the preoperative temporal bone surface using preop- erative $\mathrm{CT}$ images, and the navigation system used in the surgery room allowed the correct location marked on the patient's postauricular skin in the surgical field to be selected by the CT images. As a result, it was possible to find the flat position directly through the small incision and to shorten the operation time. All three surgeries were performed under local anesthesia and within 30 minutes. All three patients were able to terminate the operation within 30 minutes (knife to skin) and became possible under local anesthesia. The preparation time for the navigation system took within 10 minutes including the registration of the patient.

The operation time was greatly reduced by modifying the skin incision to a smaller size and by omitting the polishing of the protruding parts of the temporal bone surface. Owing to the 

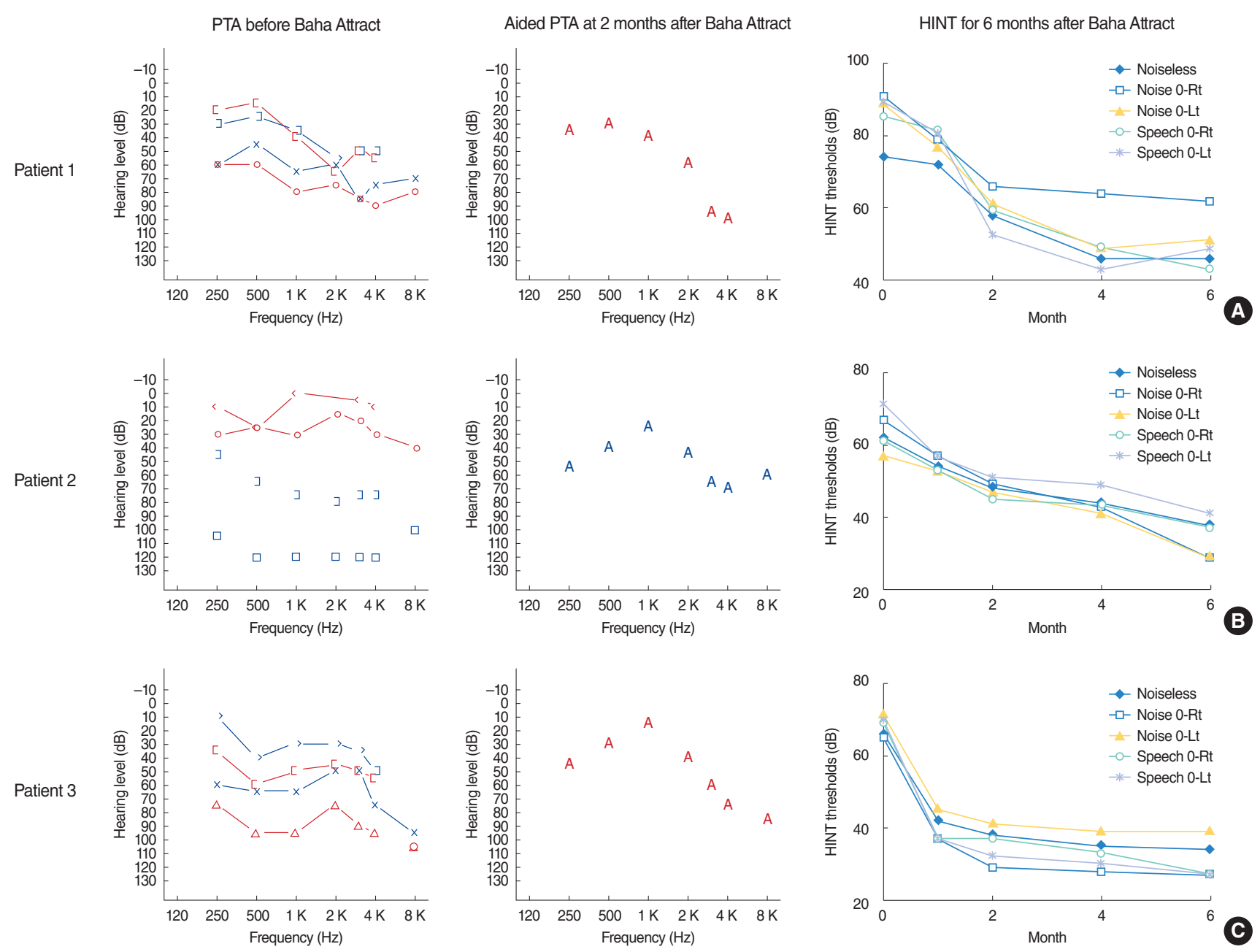

Fig. 6. Three patients who received the Baha Attract system using simplified surgery with the small incision; all scored well on the audiologic tests (sound field and Hearing in Noise Test [HINT]). Two patients with mixed hearing loss (A, C) as well as patient 2 (B), who had a type of sensorineural hearing loss, also recovered to approximately $40 \mathrm{~dB}$ on the surgical side. Compared with preoperative hearing (75, scaled out, and $90 \mathrm{~dB}$ ), the hearing gains were excellent. The HINT results showed that initial improvement was rapid, and gradual improvement continued during the 6-month follow-up. PTA, pure tone audiometry; Rt, right; Lt, left.

narrow field of view, it was necessary to first measure the skin thickness before the next step, and then to fix the magnet to prevent the surgical instruments from sticking to the magnet. Based on our experiences with three patients, we devised a simplified surgical procedure, presented in Fig. 3.

\section{Successful outcomes of surgery and audiologic function}

The stitches were removed 10 days after surgery. The external device was attached for 1 month and a hearing test was performed three times in the 6 months after surgery. No patient had complications (dizziness, pain, or wound dehiscence) immediately after surgery. All were discharged immediately and were followed for 6 months, without any wound problems or pain. Because the surgical site followed the hairline, there was no interference with hair growth, no visible scarring, and the external device could be placed in a position where a cap could be used
(Fig. 5).

The results of HINT in all patients showed that hearing continued to improve during the 6-month follow-up period. (Fig. 6). Although the three patients do not have the same hearing, the patients with mixed hearing loss showed better benefits than did the patient with sensorineural hearing loss.

\section{DISCUSSION}

A new transcutaneous bone conduction implant, the Baha Attract by the Cochlear Americas Corporation, have several benefits to patients compared to the classic penetrating Baha system [10]. In addition to the improved cosmetic effect, there were no significant differences in the understanding of quiet speech between the devices [4]. According to the company's manual, us- 
ing the indicator template, the implant should be placed generally 50-70 $\mathrm{mm}$ from the ear canal and the superior edge of the processor in line with the top of the pinna. However, since Baha Attract has no definite evidences of surgical landmarks for the appropriate positioning, the implants are placed in different locations. Different implant positioning for each patient is timeconsuming and results in unnecessary incisions. For surgeons who try to perform Baha Attract, it is necessary to have an easy and a precise surgical procedure without failure. For patients undergoing the surgery, a small incision and a short surgical time would be needed. We aimed to improve the surgical implantation of the Baha Attract. If there is sufficient anatomical evidence, this reference can be used to rapidly determine the implant position; thus, the procedure does not require a large incision, which reduces the surgical time and enables the use of local anesthesia. Therefore, the anatomical evidence presented in this study and the new surgical method based on this information provide useful information to practitioners and to patients receiving the Baha Attract.

The surgical location of the Baha Attract is the flat surface of the optimal surgical site of the temporal bone. The conditions for the magnet position of the Baha Attract are (1) a flat surface large enough for the magnet, (2) a depth of $4 \mathrm{~mm}$ or greater, and (3) easy exposure of the implantation site during surgery $[1,11,15,16]$. The safe zone, which satisfies all three conditions, can be roughly classified into two types: the conventional surgical site, where the implant is often located, and the optimal surgical site in the retrosigmoid area (Fig. 1). However, the temporal line across the conventional surgical site can cause protruding areas during surgery. Therefore, placement in the conventional surgical site requires a polishing step. According to a temporal bone thickness map [17], the bone is systematically thicker in the fronto-caudal portion. Most of the Conventional surgical site has a thickness of less than $5 \mathrm{~mm}$, but in most cases, the optimal surgical site has a thickness of more than $7 \mathrm{~mm}$. Additionally, implantation at the Conventional surgical site does not enable the wearing of hats after surgery. The anterior sigmoid sinuses are located in the optimal surgical site and the external device would contact the auricle. We set the anterior boundary of the optimal surgical site at the sigmoid sinus, and the upper boundary is bounded below the Asterion where the transverse sinus is usually located $[18,19]$. The digastric groove, the attachment point of the digastric muscle, is the lower bound. If dissection of this muscle is difficult, it becomes difficult to expose the periosteum. The posterior border is the occipitomastoid suture, which forms when the mastoid is continued at an acute angle to the occipital bone. We identified this anatomical evidence in the cadaver study and have shown that this information is useful in clinical practice for Baha Attract implant surgery.

There are three primary advantages to our novel surgical method compared to conventional surgical implantation methods for the Baha Attract. First is the cosmetic aspect. The classic
C type surgical method leaves a large scar, which may invade the inside of the hairline. However, the skin incision along the hairline showed little scarring even 6 month after surgery and did not disturb hair growth [16]. The uniform positions of the implants also enable wearing a hat. Second, we were able to omit the polishing step of the surgical procedure recommended by the company. The reason we polished the temporal bone was because of the temporal line in our analysis. When the magnet contacts the protruding part of the temporal bone, the magnitude of the vibration is disrupted, which decreases sound transmission [4,20-22]. Therefore, the position of the implant in a bump-free area can minimize exposure of the surgical window, and the surgical time can be reduced. The third benefit, the surgical time of 30 minutes (knife to skin), enabled Baha Attract implantation to be performed under local anesthesia. Depending on the proficiency of surgeons, there will be a big difference in operation time, but it is confident that most surgeons will be able to perform the surgery in a shorter time with this hair line incision than the surgery with C-type incision. Additionally, the functional and audiological results showed significant gains after implantation in patients with conductive and mixed hearing loss, as well as those with single-sided deafness.

The navigation system we used projects the anatomical spots of the temporal bone selected in the CT images directly onto the skin of the patient's temporal area. A previous study demonstrated the utility of the navigation system in Bonebridge (MedEL, Innsbruck, Austria) surgery, which is also a Baha device anchored on the temporal bone with a different figure [13]. In that study, the use of an image-guided surgical navigation system helped place the implant exactly on the simulated location of the temporal bone. The optimal surgical boundary was found manually in preoperative temporal bone $\mathrm{CT}$, and the position was arbitrarily determined using the navigation system. But the navigation system is not necessary because the navigation system is expensive and is not available in many hospitals. There was no major problem when the operation was performed by simply marking the landmarks found in the $\mathrm{CT}$ images from the external auditory canal on the skin. The navigation system was only needed for the operator's convenience, but did not affect the postoperative outcome.

In conclusion, the optimal surgical site of the temporal bone is a safe and easily accessible location for implantation of the Baha Attract. Although more clinical studies should be conducted, this newly developed surgical technique to implant the Baha Attract will provide clinicians with anatomical knowledge of the implant surgery, and patients undergoing implantation with convenience. The implants will be placed in similar positions based on anatomical evidence, so that implantation of the Baha Attract can be performed quickly, under local anesthesia, and through a small incision. 


\section{CONFLICT OF INTEREST}

No potential conflict of interest relevant to this article was reported.

\section{ACKNOWLEDGMENTS}

This research was supported by Basic Science research program through the National Research Foundation of Korea (NRF) funded by the Ministry of Education, Science and Technology (NRF2019K1A3A1A47000527) and by the Gangwon Institute for Regional Program Evaluation grant funded by the Korea Government (Ministry of Trade, Industry and Energy) (No. R0005797).

\section{ORCID}

Dong Su Jang https://orcid.org/0000-0002-1536-8110

Dong Hyo Shin https://orcid.org/0000-0003-0533-8214

Woojae Han https://orcid.org/0000-0003-1623-9676

Tae Hoon Kong https://orcid.org/0000-0002-5612-5705

Young Joon Seo https://orcid.org/0000-0002-2839-4676

\section{AUTHOR CONTRIBUTIONS}

Conceptualization: YJS. Data curation: YJS. Formal analysis: THK. Methodology: YJS, THK, DSJ. Visualization: DSJ, DHS. Writing - original draft: DSJ, WH, THK, YJS. Writing - review \& editing: WH,THK.

\section{REFERENCES}

1. Clamp PJ, Briggs RJ. The Cochlear Baha 4 Attract System: design concepts, surgical technique and early clinical results. Expert Rev Med Devices. 2015 May;12(3):223-30.

2. Hakansson B, Tjellstrom A, Rosenhall U, Carlsson P. The bone-anchored hearing aid: principal design and a psychoacoustical evaluation.Acta Otolaryngol. 1985 Sep-Oct;100(3-4):229-39.

3. House JW, Kutz JW Jr. Bone-anchored hearing aids: incidence and management of postoperative complications. Otol Neurotol. 2007 Feb;28(2):213-7.

4. Kurz A, Flynn M, Caversaccio M, Kompis M. Speech understanding with a new implant technology: a comparative study with a new nonskin penetrating Baha system. Biomed Res Int. 2014;2014: 416205.

5. Iseri M, Orhan KS, Tuncer U, Kara A, Durgut M, Guldiken Y, et al. Transcutaneous bone-anchored hearing aids versus percutaneous ones: multicenter comparative clinical study. Otol Neurotol. 2015
Jun;36(5):849-53.

6. Siegert R. Partially implantable bone conduction hearing aids without a percutaneous abutment (Otomag): technique and preliminary clinical results. Adv Otorhinolaryngol. 2011;71:41-6.

7. Siegert R, Kanderske J.A new semi-implantable transcutaneous bone conduction device: clinical, surgical, and audiologic outcomes in patients with congenital ear canal atresia. Otol Neurotol. $2013 \mathrm{Jul}$; 34(5):927-34

8. Hol MK, Nelissen RC, Agterberg MJ, Cremers CW, Snik AF. Comparison between a new implantable transcutaneous bone conductor and percutaneous bone-conduction hearing implant. Otol Neurotol. 2013 Aug;34(6):1071-5.

9. Deveze A, Rossetto S, Meller R, Sanjuan Puchol M. Switching from a percutaneous to a transcutaneous bone anchored hearing system: the utility of the fascia temporalis superficialis pedicled flap in case of skin intolerance. Eur Arch Otorhinolaryngol. 2015 Sep;272(9): 2563-9.

10. Dimitriadis PA, Farr MR,Allam A, Ray J.Three year experience with the cochlear BAHA attract implant: a systematic review of the literature. BMC Ear Nose Throat Disord. 2016 Oct;16:12.

11. Gawecki W, Stieler OM, Balcerowiak A, Komar D, Gibasiewicz R, Karlik M, et al. Surgical, functional and audiological evaluation of new Baha(®) Attract system implantations. Eur Arch Otorhinolaryngol. 2016 Oct;273(10):3123-30.

12. Morgan DE, Dirks DD, Bower DR. Suggested threshold sound pressure levels for frequency-modulated (warble) tones in the sound field. J Speech Hear Disord. 1979 Feb;44(1):37-54.

13. Kong TH, Park YA, Seo YJ. Image-guided implantation of the BonebridgeTM with a surgical navigation: a feasibility study. Int J Surg Case Rep. 2017;30:112-7.

14. Kim G, Ju HM, Lee SH, Kim HS, Kwon JA, Seo YJ. Efficacy of boneanchored hearing aids in single-sided deafness: a systematic review. Otol Neurotol. 2017 Apr;38(4):473-83.

15. Reddy-Kolanu G, Marshall A. Implantation of the Cochlear Baha(®) 4Attract system through a linear incision. Ann R Coll Surg Engl. 2016 Jul;98(6):437-8.

16. Brant JA, Gudis D, Ruckenstein MJ. Results of Baha ${ }^{\circledR}$ implantation using a small horizontal incision. Am J Otolaryngol. 2013 Nov-Dec; 34(6):641-5

17. Guignard J, Arnold A, Weisstanner C, Caversaccio M, Stieger C. A bone-thickness map as a guide for bone-anchored port implantation surgery in the temporal bone. Materials (Basel). 2013 Nov;6(11): 5291-301.

18. Gonzales MB, Hufana VD. An anatomic study of the Asterion as a landmark for the location of the transverse-sigmoid sinus junction in Filipinos. Skull Base. 2008;18(S 01):A227.

19. Ucerler H, Govsa F.Asterion as a surgical landmark for lateral cranial base approaches. J Craniomaxillofac Surg. 2006 Oct;34(7):415-20.

20. Verstraeten N, Zarowski AJ, SomersT, Riff D, Offeciers EF. Comparison of the audiologic results obtained with the bone-anchored hearing aid attached to the headband, the testband, and to the "snap" abutment. Otol Neurotol. 2009 Jan;30(1):70-5.

21. Briggs R, Van Hasselt A, Luntz M, Goycoolea M, Wigren S, Weber P, et al. Clinical performance of a new magnetic bone conduction hearing implant system: results from a prospective, multicenter, clinical investigation. Otol Neurotol. 2015 Jun;36(5):834-41.

22. Hwang JS, Kim KH, Lee JH. Factors affecting sentence-in-noise recognition for normal hearing listeners and listeners with hearing loss. J Audiol Otol. 2017 Jul;21(2):81-7. 